\title{
BI-INTERPRETABLE GROUPS AND LATTICES
}

BY

\author{
M. JAMBU-GIRAUDET
}

\begin{abstract}
A large class of 0-2 transitive lattice-ordered groups is finitely axiomatizable as a class of groups and as a class of lattices. In each model, the group structure and the lattice structure plus one parameter are bi-interpretable, sometimes up to duality only. A characterization of lattice-automorphisms of the structures is also given.
\end{abstract}

Introduction. Let $T$ be a chain, and $G$ a group of order-preserving permutations of $T$; let $(T, G)$ denote for the time being the two-sorted structure allowing us to speak of the action of $G$ on $T$ (more formal details given below). The group $G$ is partially ordered in a coordinate way and in many cases is a lattice. The aim of this paper is to interpret the two-sorted structure $(T, G)$ into the one-sorted structures at hand: in $\S 1$ we interpret under certain natural conditions $(T, G)$ into the (abstract) group $G$, in $\$ 2$ we interpret under other conditions $(T, G)$ into the lattice $G$. The interpretations use first-order formulas with at most two parameters and are of the kind routinely used in Model Theory. Obviously, each of the abstract group $G$ and of the lattice $G$ can be recovered from the two-sorted structure $(T, G)$, so combining our results yields under certain hypothesis bi-interpretability of the group structure and of thelattice structure.

In $\S 1$, we consider a class of groups $G$ previously examined in [8]. In [8] (which had in some respects been anticipated by [9]) was obtained an (not first-order) identification of the chain $T$ with a set of subgroups of $G$. We first put to good use a result of [8] to describe the action of $G$ on $T$ through the first-order properties of $G$.This approach has many advantages and yields in particular a finite set of axioms for the class of those of our groups which admit a structure of lattice-ordered groups, as well as criteria for their elementaryequivalence.

In $\S 2$, the "dual" result is obtained; it applies to a smaller class of groups which however includes the full group of order-preserving permutations of any 2-homogeneous chains: the action of $G$ on $T$, and in particular the (abstract) group structure of $G$, can be interpreted in the first-order theory of the lattice $G$, with one parameter. Exactly as before, finite axiomatization and criteria of elementary equivalence (for the involved lattices) will follow.

The program of recognizing algebraic (or topological) structures through the first-order properties of their automorphisms groups has a long history. The case of

Received by the editors January 30, 1981 and, in revised form, April 2, 1982.

1980 Mathematics Subject Classification. Primary 03C52, 20B27, 20F60; Secondary 03B10, 06A05. 
chains is discussed in [3] where some of the results of our $\$ 1$ are mentioned. Since the results of [3] allow us to recognize several chains through the first-order theory of the lattice-ordered group of all their order-preserving permutations, and, combined with some results of our $\S 1$, let the recognition carry over to the theory of the (abstract) group, we can now, with the results of our $\$ 3$, let the recognition carry over to the theory of the lattice. Typically, it follows from [3] and our $\$ 2$ :

THEOREM. If the lattice of all order-preserving permutations of a chain $T$ is elementary equivalent to the lattice of all order-preserving permutations of the real line $R$ (resp. of the rational line $Q$ ), then $T$ is isomorphic to $R$ (resp. to one of $Q$ or $R-Q$ ).

In particular, the lattices of all order-preserving permutations of the real line and of the rational line are not isomorphic. This settles an open question which was brought to my attention by W. C. Holland and A. M. W. Glass and which motivated $\S 2$ of this paper. I am grateful to them and to G. Sabbagh, my thesis advisor, for providing me with the stimulus and encouragement without which this paper could not have been written. I am especially indebted to A. M. W. Glass for checking my proofs and for correcting my english. I would like to thank the referee whose report convinced me to reshuffle the introduction.

0. Notation and preliminary remarks. If $T$ is a dense chain, let $\bar{T}$ denote its Dedekind completion. We may assume $T$ (and $\bar{T}$ ) have no greatest or least element (removing the greatest or least element of a chain does not change its 0 -permutation group).

Since $T$ is a dense subset of $\bar{T}$, any 0 -permutation of $T$ will have a unique extension to $\bar{T}$, and it will be convenient to identify such a 0 -permutation with its extension to $\bar{T}$ : a 0 -permutation of $T$ is a 0 -permutation of $\bar{T}$ which preserves $T$. With this notation, the support $S_{f}$ of a 0 -permutation $f$ of $T$ is $S_{f}=\{t \in \bar{T} ; f(t) \neq t\}$.

If $G$ is a group of 0 -permutations of $T$, hence an ordered-permutation group or 0 -p-group, we let $G T$ denote $G, e$ be the identity element of $G T$, and $G T_{+}=\{f \in$ $G T ; f>e\}$ and $G T_{-}=\{f \in G T ; f<e\}$ be the (strictly) positive and negative cones of $G T$. Examples of 0 -p-groups, which moreover are lattice-ordered permutation groups, or l-p-groups, are the following:

$A T$ : the group of all 0-permutations of $T$.

$L T$ : the group of all elements $f$ of $A T$ with $S_{f}<t$ (Left of $t$ ) for some $t$ in $T$.

$R T$ : the group of all elements $f$ of $A T$ with $S_{f}>t$ (Right of $t$ ) for some $t$ in $T$.

$B T=L T \cap R T$.

A nontrivial 0 -p-group $G T$ is said to be 0 - $n$-transitive for some positive integer $n$, or, equivalently, $G$ is said to act $0-n$-transitively on $T$, if, for any $t_{1}<t_{2}<\cdots<t_{n}$ and $t_{1}^{\prime}<t_{2}^{\prime}<\cdots<t_{n}^{\prime}$ in $T, f\left(t_{i}\right)=f\left(t_{i}^{\prime}\right)$, with $i=1,2, \ldots, n$, for some element $f$ of $G$. The chain $T$ is said to be $n$-homogeneous if $A T$ is $0-n$-transitive, or; equivalently, if some $G T$ is 0 - $n$-transitive. Clearly, any $n$-homogeneous chain, with $n \geqslant 2$, is dense and without end-points. Any 0-p-group we shall consider in this paper will be 0 - $n$-transitive for any integer $n$ and will have an element of bounded support $(G T \cap B T \neq\{e\})$. It should be kept in mind that, in order to be $0-n$-transitive for any integer $n$, it is enough for a 0 -p-group to be 0 -3-transitive, and it is enough for an $l$-p-group to be 0 -2-transitive (see [1]). 
$0-1$. Dense subsets and topology of $\bar{T}$. An orbit of $G T$ will mean an orbit of $G T$ in $\bar{T}$. Under our assumptions all orbits of $G T$ are dense in $\bar{T}$. The following are unions of orbits, hence dense subsets of $\bar{T}$ :

$T_{G L}$ : the set of greatest lower bounds (g.l.b.'s or left ends) of supports of elements of $G T \cap B T-\{e\}$.

$T_{G R}$ : the set of least upper bounds (l.u.b.'s or right ends) of supports of elements of $G T \cap B T-\{e\}$.

The interval topology of $\bar{T}$ has as a basis the set of open intervals of $\bar{T}$, and induces on any dense subset its interval topology.

Let $g$ be any element of $G T \cap B T-\{e\}$, then:

(i) For any open interval $\dot{I}$ of $\bar{T}$ and any $t$ in $\bar{T}$, there is some conjugate $f$ of $g$ in $G T$ with $t \in S_{f} \subseteq \dot{I}$.

(ii) For any conjugate $f$ of $g$ in $G T$ and any $t \in S_{f}$, there is some open interval $\dot{I}$ of $\bar{T}$ with $t \in \dot{I} \subseteq S_{f}$.

Hence the set of supports of conjugates of $g$ in $G T$ is a basis for the interval topology of $\bar{T}$, inducing a basis for the interval topology on any dense subset of $\bar{T}$.

This also holds for the set of supports of any class of elements of $G T$ intersecting $B T-\{e\}$ and closed by conjugacy, such as $G T$ or any other class intersecting $B T-\{e\}$ and definable in the lattice ordered group language.

$0-2$. Full structures and dualities. Let $S$ be a union of orbits of $G T$. Note that the action of $G$ on $S$ is not 1-transitive in general (except if $S$ is one orbit of $G T$ ) although it is on $T$.

The full structure $G T \cup S$ has as a domain the set $G T \cup S$ and as predicates:

(i) a one-placed predicate discriminating $G T$ from $S$,

(ii) a two-placed predicate interpreted in $S$ by the order induced on $S$ from $\bar{T}$,

(iii) a three-placed predicate interpreting the action of $G T$ on $S$, i.e. satisfied by those $\left(f, t_{1}, t_{2}\right)$ of $G T \times S^{2}$ such that $f\left(t_{1}\right)=t_{2}$.

Clearly such a structure uniquely determines the order of $\bar{T}$ and the action of $G T$ on $\bar{T}$, hence the ordered-group structure of $G T$ is definable in it. Intersections of supports of elements of $G T$ with $S$ and orbits of $G T$ included in $S$ are also definable, now with a parameter.

For predicates (i) (order of $S$ ) and (ii) (action) we can define dual predicates in a natural way. We shall further on be interested in (1) duality of (i), and (2) duality of both (i) and (ii):

(1) The order-dual full structure $(G T)^{*} \cup S^{*}$ has the same domain as $G T \cup S$ and the same interpretation for $S, G T$, and action of $G T$ on $S$ ((i) and (iii)), but predicate (ii) is now interpreted by the dual order of $S$. In this structure, the same formulas which defined the ordered group structure of $G T$ now define the same group structure with the dual order.

Hence $(G T)^{*}$ denotes the group $G T$ with its dual order.

(2) The group-dual full structure $G_{*} T \cup S^{*}$ has the same domain as $G T \cup S$ and the same interpretation for $S$ and $G T$ (i) but:

-predicate (ii) is interpreted by the dual order of $S$,

-predicate (iii) is interpreted by the dual action of $G T$ on $S$, i.e. by those $\left(f, t_{1}, t_{2}\right)$ of $G T \times S^{2}$ such that: $f\left(t_{2}\right)=t_{1}$ (or equivalently $f^{-1}\left(t_{1}\right)=t_{2}$ ). 
In this structure, the formulas which defined the ordered-groupstructure of $G T$ now define the same order with the dual group structure, i.e.: the group structure with product denoted by * and defined for any $f$ and $g$ in $G T$ by

$$
f * g=g f=\left(f^{-1} g^{-1}\right)^{-1} \text {. }
$$

Hence $G_{*} T$ denotes the ordered set $G T$ with its dual group structure.

0-3. The importance of parameters. Let $M_{1}$ be some model of a language $L_{1}$ and $M_{2}$ a model of some language $L_{2}$. Let $C$ be a finite set of constants (parameters) interpreted in $M_{1}$ and take formulas of $L_{1} \cup C$ denoted:

$D(u)$, defining a subset of $M_{1}$ called the domain of interpretation of $M_{2}$.

$E(u, v)$, defining a relation of $M_{1}$ which is an equivalence on the domain of interpretation of $M_{2}$ and is called the interpretation of the equality relation of $M_{2}$.

$F\left(u_{1}, u_{2}, \ldots, u_{n_{i}}\right)$, for each predicate $P_{i}$ with arity $n_{i}$ in $L_{2}$, called the interpretation of $P_{i}$.

The formulas $D, E, F_{i}$ are said to interpret $M_{2}$ in $M_{1}$ if the quotient set $D / E$ with predicates induced by the $F_{i}$ 's is isomorphic to $M_{2}$. Note that an interpretation without parameters $(C=\varnothing)$ should stay unchanged through all automorphisms of $M_{1}$.

Clearly if some full structure $G T \cup S$ is interpreted in some other structure, so is the lattice-ordered group $G T$. Our purpose being to interpret full structures in groups and lattices, we must be aware of the following limitations.

REMARK 1. For any $g$ in a lattice - ordered group $G$, the map $g \mapsto f g$ is a lattice-automorphism and not (if $g \neq e$ ) a group-automorphism. So, when interpreting with the language of lattices in $G$, we will always need a parameter to choose among different copies of the group structure.

REMARK 2. The chain $T$ may have an anti-order-automorphism $\dot{I}$ : this is the case for any chain underlying a totally ordered field, where the inverse function reverses order. (Such a chain is 2-homogeneous, hence of present interest. See also [5] for other examples.) In such a case:

(a) The structures $A T$ and $B T$ have a group-automorphism: $f \mapsto \dot{I} f \dot{I}^{-1}$, which is an anti-order-automorphism. So, when interpreting in the language of groups in $A T$ and $B T$, we will need a parameter to choose the order of $A T$ and $B T$ up to duality.

(b) The structures $A T$ and $B T$ have a lattice-automorphism: $f \mapsto I f^{-1} \dot{I}^{-1}$, fixing $e$, which is an "anti"-group-automorphism. When interpreting in the language of lattices plus one parameter (see Remark 1) in $A T$ and $B T$, we will need a second parameter to choose the group structures of $A T$ and $B T$ up to duality.

However, in some other cases, the parameters described in (a) and (b) will not be necessary (see Corollaries 1-3(2) and 2-2(2) in this paper and [6 or 7]).

REMARK 3. If $S$ is some orbit of $G T$ in $\bar{T}$, it may happen that $A T$ and $A S$ are equal (as sets of 0 -permutations of $\bar{T}$ ). Moreover, there may be some 0 -isomorphism between $S$ and $T$ inducing, through conjugacy, the identity on $A T=A S$ (see [4]). In such a case, even when interpreting with the language of both groups and orders in $G T$, we will need a parameter to choose $T$ from other orbits.

This has, as trivial (but not unique) exceptions, groups $G T$, where $G T$ has one orbit in $T, T$ being the real line or a long line for instance, and $G T$ being one of $A T$, 
$L T, R T, B T$, the subgroup of almost everywhere differentiable functions of one of those the subgroup of piecewise linear functions of one of those when $T$ is the real line, etc.... (See [5 or 6] for examples with more than one orbit.)

1. Interpreting in groups. The following lemma is a basic tool for our construction. Its proof is implicit in [8].

LEMMA (MCCLEARY). For any 0-3 transitive 0-p-group GT with a positive element of bounded support, and any elements $p, f$ and $g$ of $G T-\{e\}$, the formula $F_{0}(p, f, g)$ of the group language

$$
F_{0}(p, f, g) \leftrightarrow \forall u\left(\text { f and } u p u^{-1} g u p^{-1} u^{-1} \text { commute }\right)
$$

is true in GT if and only if one of the following holds:

(1) $p \in G T_{+}$and $S_{f}<S_{g}$,

(2) $p \in G T_{-}$and $S_{g}<S_{f}$.

Let $f<_{p} g$ denote the formula $F_{0}(p, f, g)$ of the lemma.

What [8] actually states is that, for any $p \neq e$, the formula " $\exists u \neq e \exists v \neq e$ $\left(u<_{p} v\right)$ " is equivalent to: " $p \in G T_{+} \cup G T_{-}$" (and so, $G T_{+} \cup G T_{-}$is definable with the language of groups). But the reader will have no difficulty in seeing that the lemma follows from the proof given there (see proof of Theorem 4, p. 507). More explicit proofs can be found in [5 and $\mathbf{1}$ ].

REMARK. From McCleary's lemma it is clear that $G T \cap B T$ is defined in $G T$ by the formula

$$
\exists v \exists w \exists w^{\prime}\left(S_{w}<_{v} S_{u} \wedge S_{u}<_{v} S_{w^{\prime}}\right)
$$

in the group language without any parameter.

In a similar way, $G T \cap L T$ and $G T \cap R T$ are definable in the group language plus one parameter interpreted in $G T_{+} \cup G T_{-}$.

THEOREM 1. Let GT be a 0-3 transitive 0-p-group with a positive element of bounded support (and remember $G T_{+} \cup G T_{-}$is definable in the language of groups without parameters).

There are formulas of the group language plus one parameter $\tilde{p}$ which interpret in GT one of the full structures:

(1) $G T \cup T_{G L}$, if parameter $\tilde{p}$ is interpreted by some $p$ in $G T_{+}$.

(2) $(G T)^{*} \cup T_{G R}^{*}$, if parameter $\tilde{p}$ is interpreted by some $p$ in $G T_{-}$.

The order dual of a full structure being obviously bi-interpretable with it, we have

COROLlaRY 1-1. If GT is a 0-3 transitive 0-p-group with a positive element of bounded support, the full structures $G T \cup T_{G L}$, and $G T \cup T_{G R}$ can be interpreted in $G T$ with the language of ordered groups.

Moreover, since $G T_{+} \cup G T_{-}$is definable in the language of groups, we deduce from Theorem 1

COROLlARY 1-2. Let $F$ be a formula of the language of full structures and GT a 0-3 transitive 0-p-group with a positive element of bounded support, then any of:

(1) $F$ is satisfied in both $G T \cup T_{G L}$ and $(G T)^{*} \cup T_{G R}^{*}$, 
(2) $F$ is satisfied in one of $G T \cup T_{G L}$ or $(G T)^{*} \cup T_{G R}^{*}$ is equivalent to the satisfaction of a formula of the language of groups in GT. This still holds if $F$ has parameters in GT.

COROLlaRY 1-3. Let GT be a 0-3 transitive 0-p-group with a positive element of bounded support. Then:

(1) The following conditions are equivalent:

(i) The order of GT is definable in the language of groups.

(ii) $G T$ and $(G T)^{*}$ are not elementary equivalent ordered-groups.

(iii) The full structures $G T \cup T_{G L}$ and $(G T)^{*} \cup T_{G R}^{*}$ are not elementary equivalent.

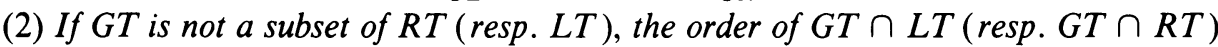
is definable in the language of groups without a parameter.

COROLlARY 1-4. Let GT and HS be 0-3 transitive 0-p-groups with positive elements of bounded support.

(1) The following conditions are equivalent:

(i) GT and HS are elementary equivalent as groups.

(ii) One of the ordered groups $G T$ and $(G T)^{*}$ is elementary equivalent to the ordered group $H S$.

(iii) One of the full structures $G T \cup T_{G L}$ and $(G T)^{*} \cup T_{G R}^{*}$ is elementary equivalent to the full structure $H S \cup S_{H L}$.

(2) If GT is not a subset of BT (resp. LT, resp. RT), then GT and GT $\cap B T$ (resp. $G T \cap L T$, resp. $G T \cap R T$ ) are not elementary equivalent as groups.

Assuming Theorem 1 for the moment, let us deduce from it and from the definability of $G T_{+} \cup G T_{-}$in the group $G T$ Corollaries 1-2 to 1-4.

First note that, if $F$ is any formula of the full structures language and $p$ any element of $G T_{+} \cup G T_{-}$, we can, using Theorem 1, construct a formula $F^{\prime}(p)$ of the group language plus parameter $p$ such that:

$$
\begin{gathered}
G T \cup T_{G L} \vDash F \leftrightarrow F^{\prime}(p) \quad \text { whenever } p>e, \\
(G T)^{*} \cup T_{G R}^{*}=F \leftrightarrow F^{\prime}(p) \quad \text { whenever } p<e .
\end{gathered}
$$

We shall use this notation $F^{\prime}(p)$ throughout the proofs.

Proofs of Corollaries 1-2 To 1-4. 1-2: (1) is equivalent to: $\forall u\left(u \in G T_{+} \cup G T_{-}\right.$ $\rightarrow F^{\prime}(u)$ ), and (2) is equivalent to: $\exists u\left(u \in G T_{+} \cup G T_{-} \wedge F^{\prime}(u)\right)$ which are both formulas of the language of groups.

1-3(1): (i) $\rightarrow$ (ii) $\rightarrow$ (iii) is trivial. Assuming (iii), let $F$ be a formula of the full structure language which is satisfied in $G T \cup T_{G L}$ and not in $(G T)^{*} \cup T_{G R}^{*}$. Then

$$
\begin{gathered}
G T \cup T_{G L} \vDash F^{\prime}(p) \text { when } p>e, \\
(G T)^{*} \cup T_{G R}^{*} \vDash \neg F^{\prime}(p) \text { when } p<e .
\end{gathered}
$$

So, for any $p$ in $G T_{+} \cup G T_{-}, G T \vDash F^{\prime}(p)$ iff $p>e$, and " $u \in G T_{+} \cup G T_{-} \wedge F^{\prime}(u)$ " defines $G T_{+}$in $G T$ with the group language.

1-3(2): If $G T$ is not a subset of $R T$, the formula of the language of full structures

$$
\exists u \forall v \neg\left(S_{v}<S_{u}\right)
$$


is satisfied in $(G T \cap L T) \cup T_{G L}$, since $G T \cap L T$ is not a subset of $R T$, but is not sattsfied in $(G T \cap L T)^{*} \cup T_{G R}^{*}$, where $(G T \cap L T)^{*}=(G T)^{*} \cap R\left(T^{*}\right)$. Hence $(G T$ $\cap L T$ ) satisfies (iii) of 1-3(1).

The rest of the proof is similar.

1-4(1): Clearly (iii) $\rightarrow$ (ii) $\rightarrow$ (i). Assume (iii) is false and let $F_{1}$ and $F_{2}$ be formulas of the language of full structures such that $G T \cup T_{G L} \vDash F_{1}, G T^{*} \cup T_{G R}^{*} \vDash F_{2}$ and $H S \cup S_{H L} \vDash \neg F_{1} \wedge \neg F_{2}$.

Using only the group language we get from 1-2

$$
G T \vDash \forall u\left(u \in G T_{+} \cup G T_{-} \rightarrow\left(F_{1}^{\prime}(u) \vee F_{2}^{\prime}(u)\right)\right)
$$

and

$$
H S \vDash \exists u\left(u \in H S_{+} \cup H S_{-} \wedge \neg F_{1}^{\prime}(u) \wedge \neg F_{2}^{\prime}(u)\right)
$$

where $u \in G T_{+} \cup G T_{-}$and $u \in H S_{+} \cup H S_{-}$are equivalent to the same groupformula, so this contradicts (i).

1-4(2): If $G T$ is not a subset of $B T$, then the formula of the full structures language

$$
\exists u \forall v \neg\left(S_{u}<S_{v}\right) \vee \exists u \forall v \neg\left(S_{v}<S_{u}\right)
$$

is satisfied in $G T \cup T_{G L}$ but not in $(G T \cap B T) \cup T_{G L}$ and $(G T \cap B T)^{*} \cup T_{G R}^{*}$ (where $T_{G L}=T_{G \cap B, L}$ and dually). The conclusion follows from 1-4(1).

The rest of the proof is similar.

Proof of Theorem 1. Let $\tilde{p}$ be interpreted by some $p \in G T_{+}$and let $D$ be the group-formula defining $G T \cap B T-\{e\}$ in $G T$ without a parameter.

Let $O(u, v)$ be the formula:

$$
O(u, v) \leftrightarrow \forall w\left(S_{u}<S_{w}\right)^{\prime}(p) \rightarrow\left(S_{v}<S_{w}\right)^{\prime}(p)
$$

of the group language plus one parameter $p$.

Let $E(u, v)$ be the formula:

$$
E(u, v) \leftrightarrow O(u, v) \wedge O(v, u) .
$$

Clearly, for any $f, g \in G T \cap B T-\{e\}, O(f, g)$ is satisfied iff the l.u.b. of $S_{f}$ in $\bar{T}$ is more than the l.u.b. of $S_{g}$, and $E(f, g)$ iff those l.u.b.'s are equal. The quotient set $D / E$ with predicate 0 is isomorphic to the chain $T_{G L}$, the natural isomorphism $I$ between them being such that, for any $s$ in $T_{G L}, I(s)$ is the set of all elements $f$ of $G T \cap B T-\{e\}$ such that $s$ is the l.u.b. of $S_{f}$ in $\bar{T}$.

Now let $A(u, v, w)$ be the formula $A(u, v, w) \leftrightarrow E\left(u v u^{-1}, w\right)$. This interprets the action of $G T$ on $T_{G L}$ since, for any $(f, t, s) \in G T \times S^{2}, g \in I(s)$, and $h \in I(t)$, we have $S_{f g f^{-1}}=f\left(S_{g}\right)$, hence $f g f^{-1} \in I(f(S))$, and $A(f, g, h)$ is satisfied in $G T$ iff $f g f^{-1} \in I(t)$.

Clearly, if $\tilde{p}$ is interpreted by $p \in G T_{-}$, these same formulas will interpret the full structure $G T^{*} \cup T_{G R}^{*}$ where $T_{G R}$ is the set of all g.l.b.'s of elements of $G T \cap B T$ in $\bar{T}$.

Theorem 1 is proved. 
COROLlARY 1-5. If GT and HS are 0-3 transitive 0-p-groups with positive elements of bounded support such that:

(i) Every element of $\bar{T}$ is a l.u.b. and a g.l.b. for supports of elements of GT, i.e.: $\bar{T}=T_{G L}=T_{G R}$.

(ii) Every element of $\bar{S}$ is a l.u.b. and a g.l.b. for supports of elements of HS, i.e.: $\bar{S}=S_{G L}=S_{G R}$. $\bar{S}$.

(iii) For some integer $n, G T$ has at least $n$ orbits in $\bar{T}$ and HS has less than $n$ orbits in

Then GT and HS are not elementary equivalent groups.

Proof. It is clear from the proof of Theorem 1 that, under assumption (i), the full structure $G T \cup \bar{T}$ can be interpreted in the group $G T$.

So, for any integer $n$, the satisfaction of the formula of the language of full structures

\section{"GT has $n$ orbits in $\bar{T}$ "}

is equivalent to the satisfaction of a formula $F$ of the language of ordered groups in $G T$.

Obviously $F$ is satisfied in $G T$ if and only if it is satisfied in $(G T)^{*}$. It follows then from Corollary 1-2 that $F$ is a formula of the group language.

Now, under assumption (ii), $F$ is satisfied in the group $H S$ iff $H S$ has $n$ orbits in $\bar{S}$, and the conclusion follows from (iii).

The following result was proved in [8] using considerable knowledge of the maximal subgroups of the structures. Our construction yields a simpler proof.

Corollary 1-6 (MCClEARY). Let GT and HS be 0-3 transitive 0-p-groups with positive elements of bounded support; then any group isomorphism $\psi$ from GT onto HS is of the form

$$
\psi(f)=\theta f \theta^{-1} \quad \text { for any } f \text { in } G T
$$

where $\theta$ is an order or an anti-order isomorphism from Tonto some orbit of HS in $\bar{S}$.

Proof. Let $\psi$ be a group isomorphism between $G T$ and $H S$ as in Corollary 1-6 and let $p$ be an element of $G T_{+}$. Since $G T_{+} \cup G T_{-}$and $H S_{+} \cup H S_{-}$are defined by the same formula of groups in $G T$ and $H S$ respectively, $\psi(p)$ is in $H S_{+} \cup H S_{-}$.

Formulas of the group language plus one parameter interpreted in $G T$ by $p$ interpret $T_{G L}$ in $G T$ (Theorem 1).

The same formulas plus one parameter interpreted in $H S$ by $\psi(p)$ will interpret in $H S$ one of $S_{H L}$ (if $\psi(p)$ is in $H S_{+}$) and $S_{H R}^{*}$ (if $\psi(p)$ is in $H S_{-}$).

Any element of those interpretations is a class of elements, and we let $t_{g, q}$ denote it when $g$ belongs to it and $q$ interprets the parameter in the corresponding interpretation.

We can see from the interpretation of action in the proof of Theorem 1, that, if $f, g$ and $q$ are all in $G T$ or all in $H S, f\left(t_{g, q}\right)=t_{f g f^{-1}, q}$.

Now, for any $t_{g, p}$ in the interpretation of $T_{G L}$, let $\theta$ be the map such that $\theta\left(t_{g, p}\right)=s_{\psi(g), \psi(p)}$. 
$\boldsymbol{\theta}$ induces an order isomorphism from $T$ onto some orbit of $H S$ in $\bar{S}$, if $\psi(p)$ is in $H S_{+}$, from $T$ onto some orbit of $H S$ in $\bar{S}^{*}$, if $\psi(p)$ is in $H S_{-}$.

It is therefore as described in Corollary 1-6.

Now, for any $f$ in $G T_{+}$and $g$ in $H S \cap B S-\{e\}$,

$$
\begin{aligned}
\psi(f)\left(s_{g, \psi(p)}\right) & =s_{\psi(f) g(\psi(f))^{-1}, \psi(p)}=s_{\psi\left(f \psi^{-1}(g) f^{-1}\right), \psi(p)}=\theta\left(t_{f \psi^{-1}(g) f^{-1}, p}\right) \\
& =\theta f\left(t_{\psi^{-1}(g), p}\right)=\theta f \theta^{-1}\left(s_{g, \psi^{-1}(p)}\right)
\end{aligned}
$$

which completes the proof.

Note that, if $G T$ is a 0-2 transitive l-p-group with an element of bounded support (and hence satisfies the hypothesis of Theorem 1), it is 0-2 transitive on any of its orbits (see [1]). So, if we let:

$P(u)$ denote the formula which defines $\left(G T_{+} \cup G T_{-}\right) \cap B T$ in any $G T$,

$(S(u, p), O(u, v, p))$ be the formulas which interpret the ordered orbit of the l.u.b. of $S_{p}$ in $T_{G L}$ when $p \in G T_{+}$and the ordered orbit of the g.l.b. of $S_{p}$ in $T_{G R}^{*}$ when $p \in G T$, then, any group $G$ which admits a structure of a $0-2$ transitive l-p-group with an element of bounded support satisfies a formula of the language of groups which can be roughly described as follows.

"For some $p$ satisfying $P(u)$, the set defined by $S(u, p)$ is totally ordered by the formula $O(u, v, p)$ and conjugacy defines a 0-2 transitive faithful action of $G$ on the chain $(S(u, p), O(u, v, p))$ such that the pointwise induced order on $G$ is a lattice order."

Conversely, any group satisfying this formula will be a $0-2$ transitive $l$-group of 0 -permutations of the chain we shall be able to interpret in it, and will have an element of bounded support.

So we have proved the following

COROLlARY 1-7. The class of groups which admit a structure of a 0-2 transitive l-p-group with an element of bounded support is the class of models of one formula.

From Corollary 1-2, if $F$ is any formula of the language of full structures, the two classes of groups $G T$ which admit a structure of a 0-2 transitive $l$-p-group with an element of bounded support and are such that:

(1) $F$ is satisfied in both $G T \cup T_{G L}$ and $(G T)^{*} \cup T_{G R}^{*}$ or

(2) $F$ is satisfied in one of $G T \cup T_{G L}$ or $(G T)^{*} \cup T_{G R}^{*}$ are also classes of models of one formula.

\section{Interpreting in lattices.}

DeFinition. An element $f$ of an $l$-p-group $G T$ is said to be an $l$-prime of $G T$, or an $l$-prime, if $G T$ contains no proper restriction of $f$, that is, a $g \in G T$ different from $e$ and $f$ such that at each point of $T g$ agrees with $f$ or with $e$.

Remember a 0-2 transitive $l$-p-group with an element of bounded support satisfies the hypothesis of Theorem 1. The fact that some element of bounded support should be $l$-prime seems to be an even stronger condition. 
THEOREM 2. If GT is a 0-2 transitive l-p-group with an l-prime of bounded support, we can find:

Two subsets $P_{1}$ and $P_{2}$ of $G T$ such that $P=P_{1} \cup P_{2}$ is definable in the language of lattices plus one parameter $\tilde{e}$. ( $P_{1}$ and $P_{2}$ generally not definable.)

Formulas of the language of lattices plus two parameters $\tilde{e}$ and $\tilde{p}$ which, whatever the interpretation of $\tilde{e}$, hence of $P$, is, interpret in $G T$ one of the full structures:

(1) $G T \cup T_{G L}$ if parameter $\tilde{p}$ is interpreted in $P_{1}$,

(2) $G_{*} T \cup T_{G R}^{*}$ if parameter $\tilde{p}$ is interpreted in $P_{2}$.

This theorem, if we consider the lattice theory plus one parameter, is a dual result to Theorem 1. If moreover we remember that all elements of $G T$ have the same one-type in the lattice theory, hence that every lattice formula with one parameter is equivalent to a lattice formula without parameter, we get similar corollaries to those of Theorem 1, with similar proofs. The proofs will be left to the reader.

COROLLARY 2-1 (SEE 1-2). Let $F$ be a formula of the language of full structures and GT a 0-2 transitive l-p-group with an l-prime element of bounded support. Then any of:

(1) $F$ is satisfied in both $G T \cup T_{G L}$ and $G_{*} T \cup T_{G R}^{*}$,

(2) $F$ is satisfied in one of $G T \cup T_{G L}$ and $G_{*} T \cup T_{G L}^{*}$

is equivalent to the satisfaction of a formula of the language of lattices in GT.

COROLlARY 2-2 (SEE 1-3). Let GT be a 0-2 transitive l-p-group with an l-prime element of bounded support. Then:

(1) The following conditions are equivalent:

(i) The group structure of GT is definable in the language of lattices plus one parameter.

(ii) The order of GT is definable in the language of groups.

(2) If GT is not a subset of $R T$ (resp. LT), then GT $\cap L T$ (resp. GT $\cap R T$ ) satisfy (i) of (1).

COROLlARY 2-3 (SEE 1-4). If GT and HS are 0-2 transitive l-p-groups with l-prime elements of bounded support, the following are equivalent:

(1) One of GT and $G T^{*}$ is elementary equivalent to $H S$ as a lattice.

(2) One of GT and $G_{*} T$ is elementary equivalent to $H S$ as a group.

Proof of Theorem 2. Let GT satisfy the hypothesis of Theorem 2.

$L$ will denote the language of lattices including symbols $\vee$ and $\wedge$ for supremum and infimum, and, if necessary, symbols $\leqslant,<, G T_{+}$and $\overline{G T_{-}}$.

Let, for the moment, the parameter $\tilde{e}$ be interpreted by the identity.

Fact 1. The set of $l$-primes of $G T$ is definable with language $L \cup\{\tilde{e}\}$.

Proof. For any $f \neq e$ in $G T$ and not in $G T_{+} \cup G T_{-}, f \vee e$ is a proper restriction of $f$. Hence every l-prime of $G T$ must be in $G T_{+} \cup G T_{-}$. For symmetry reasons, it is enough to define $l$-primes which are in $G T_{+}$.

For any $f$ and $g$ in $G T_{+}$, if $g$ is a proper restriction of $f$, so is $f g^{-1}$. Hence, " $g$ is a proper restriction of $f$ " is equivalent to

$$
g \neq e \wedge \exists u \neq e(u \wedge g=e \wedge u \underline{\vee} g=f)
$$

and Fact 1 is clear (even for arbitrary $l$-p-groups). 
It will be useful to remember that, as a trivial consequence of Fact 1 , every conjugate of an $l$-prime is an $l$-prime.

Definition. For any $f \neq e$ in $G T$, let $\bar{S}_{f}$ denote the closure of $S_{f}$ for the interval topology of $\bar{T}$.

Fact 2. For any $f$ and $g$, both in $G T_{+}$or both in $G T_{-}, S_{f} \subseteq \bar{S}_{g}$ is a formula of $L \cup\{\tilde{e}\}$.

Proof. Let $f$ and $g$ be both in $G T_{+}$(the other case is dual).

Since open intervals and supports of positive elements are bases for the same topology in $\bar{T}, S_{f} \subseteq \bar{S}_{g}$ is equivalent to

$$
\forall u \in G T_{+}\left(S_{u} \cap S_{g}=\varnothing \rightarrow S_{u} \cap S_{f}=\varnothing\right)
$$

and, for $f$ and $g$ both in $G T_{+}$, this is equivalent to

$$
\forall u(u \wedge g=e \rightarrow u \wedge f=e)
$$

which is a formula of $L \cup\{\tilde{e}\}$.

Fact 3. If $f$ is an l-prime of $G T$ and $S_{f}$ is bounded, then $\bar{S}_{f}$ is a convex subset of $\bar{T}$.

It is enough to prove Fact 3 when $f$ is in $G T_{+}$.

Assume $S_{f}$ is bounded and $\bar{S}_{f}$ is not a convex subset of $\bar{T}$. We can find $t_{1}<t<t_{2}<t_{3}<t^{\prime}$ in $T$ with $t_{1}<S_{f}, t \in S_{f},\left[t_{2}, t_{3}\right] \cap S_{f}=\varnothing$ and $t^{\prime} \in S_{f}$.

Let $\alpha \in A T$ be the restriction of $f$ to $\left[t_{1}, t_{2}\right]$. Since $\alpha(t)=f(t) \neq t, \alpha$ is not $e$, and since $\alpha\left(t^{\prime}\right)=t^{\prime} \neq f\left(t^{\prime}\right), \alpha$ is not $f$.

We want to prove that $\alpha$ is an element of $G T$ and, hence, that $f$ is not $l$-prime.

Take $t_{1}^{\prime}<f\left(t_{1}^{\prime}\right)=t_{2}^{\prime}<t_{3}^{\prime}$ in $T$, with $t_{1}^{\prime} \in S_{f}$ and $t_{3}^{\prime}>S_{f}$.

Since $G T$ is 0-3 transitive, we can find $g$ in $G T$ such that

$$
g\left(t_{1}^{\prime}\right)=t_{1}, \quad g\left(t_{2}^{\prime}\right)=t_{2} \text { and } g\left(t_{3}^{\prime}\right)=t_{3} .
$$

Since $S_{\alpha} \subseteq\left[t_{1}, t_{2}\right]$, we have, for any $s$ in $S_{\alpha}, \alpha(s)=f(s) \leqslant f\left(t_{2}\right)=t_{2}$ and

$$
g f g^{-1}(s) \geqslant g f^{-1} g^{-1}\left(t_{1}\right)=g f\left(t_{1}^{\prime}\right)=g\left(t_{2}^{\prime}\right)=t_{2} .
$$

Hence $\alpha \leqslant g f g^{-1}$. Besides,

$$
\left.S_{f \wedge g f g^{-1}}=S_{f} \cap g\left(S_{f}\right) \subseteq(] t_{1}, t_{2}[\cup] t_{3}, t_{3}^{\prime}[) \cap\left[g\left(t_{1}^{\prime}\right), g\left(t_{3}^{\prime}\right)\right]=\right] t_{1}, t_{2}[
$$

where $\left.S_{f} \cap\right] t_{1}, t_{2}\left[=S_{\alpha}\right.$. So $S_{f \wedge g f g^{-1}}=S_{\alpha}$. Since $\alpha$ is a restriction of $f$, we get

$$
f \wedge g f g^{-1}=\alpha \wedge g f g^{-1}
$$

and we have proved above that $\alpha \leqslant g f g^{-1}$. So $\alpha=f \wedge g f g^{-1}$ where $f$ and $g$ are in $G T$. So $\alpha$ belongs to $G T$.

Definition. For any $f, g$ and $h$ in $G T-\{e\}, S_{g}$ is between $S_{f}$ and $S_{h}$ if either $S_{f}<S_{g}<S_{h}$ or $S_{h}<S_{g}<S_{h}$ holds.

Fact 4. For $f, g$ and $h$, all in $G T_{+}$or all in $G T_{-}$, " $S_{g}$ is between $S_{f}$ and $S_{h}$ " is a formula $L \cup\{\tilde{e}\}$.

Proof. Let $f, g$ and $h$ be all in $G T_{+}$(the other case is dual).

If $S_{g}$ is between $S_{f}$ and $S_{h}$, the formula of $L \cup\{\tilde{e}\}$

$$
\forall u\left(u \text { is } l \text {-prime } \wedge u \wedge f \neq e \wedge u \wedge h \neq e \rightarrow S_{g} \subseteq \bar{S}_{u}\right)
$$

is satisfied, as a consequence of Facts 1,2 and 3. 
The converse follows from Fact 1:

Assume $S_{g}$ is not between $S_{f}$ and $S_{h}$ and take $t_{1} \in S_{f}, t_{2} \in S_{g}$ and $t_{3} \in S_{h}$ with $t_{2} \notin\left[t_{1}, t_{3}\right]$.

Since $G T$ is 0-3 transitive, we can find a conjugate $u$ of an $l$-prime of bounded support, hence $u$ an $l$-prime (Fact 1) such that $\left[t_{1}, t_{3}\right] \subseteq S_{u}$ and $t_{2} \notin \bar{S}_{u}$. Hence the above formula is not satisfied.

Fact 5. GT $\cap B T$ is definable with language $L \cup\{\tilde{e}\}$.

Proof. For any $f$ in $G T_{+}$, " $f \in G T \cap B T$ " is equivalent to $\exists u \exists v\left(S_{f}\right.$ is between $S_{u}$ and $S_{v}$ ) which is a formula of $L \cup\{\tilde{e}\}$ (Fact 4).

The dual fact holds for $f \in G T_{\text {. }}$.

So, for any $f$ in $G T-\{e\}$, " $f \in G T \cap B T$ " is equivalent to $f \vee e \in G T \cap B T \wedge f$ $\wedge e \in G T \cap B T$.

Fact 6. For any elements $f$ and $g$ and any $l$-prime elements $a$ and $b$, all in $G T_{+} \cap B T$ or all in $G T_{-} \cap B T$,

$$
\text { " } S_{a}<S_{b} \leftrightarrow S_{f}<S_{g} "
$$

is a formula of $L \cup\{\tilde{e}\}$. We let $S_{f}<_{a, b} S_{g}$ denote it.

Proof. Take $a$ and $b$ in $G T_{+} \cap B T$. Since $\bar{S}_{a}$ and $\bar{S}_{b}$ are convex (Fact 3), " $S_{a}<S_{b}$ or $S_{b}<S_{a}$ " is equivalent to $a \wedge b=e$.

Assume $S_{a}<S_{b}$ and take $f$ and $g$ in $G T_{+} \cap B T$.

If they satisfy the formula $F(a, b, f, g)$ of $L \cup\{\tilde{e}\}$

$$
F(a, b, f, g) \leftrightarrow S_{b} \text { is between } S_{a} \text { and } S_{f} \wedge S_{f} \text { is between } S_{b} \text { and } S_{g}
$$

then we have $S_{f}<S_{g}$.

So, $a, b, f, g$ being all elements of bounded support, $S_{f}<S_{g}$ is equivalent to

$\exists u, v, l$-prime elements of $G T_{+} \cap B T(F(a, b, u, v) \wedge F(u, v, f, g) \wedge u \wedge v=e)$,

which is a formula of $L \cup\{e\}$.

Clearly, if $S_{b}<S_{a}$, the same formula is equivalent to $S_{g}<S_{f}$. The case when $a, b, f, g$ are all in $G T_{-}$is similar.

Notations. For any $f$ in $G T \cap B T-\{e\}$, let $l_{f}$ be the g.l.b. (left end) of $S_{f}$ in $\bar{T}$ and $r_{f}$ the l.u.b. (right end) of $S_{f}$ in $\bar{T}$.

For any $l$-prime element $f$ in $G T \cap B T$, let $\stackrel{\circ}{S_{f}}$ be $\bar{S}_{f}$ without its end points $l_{f}$ and $r_{f}$.

Fact 7. (a) For any $f, g, h l$-primes in $B T$, " $S_{g}$ is between $S_{f}$ and $S_{h}$ " is a formula of $L \cup\{e\}$.

(b) Let $\tilde{a}, \tilde{b}, \tilde{a}^{\prime}, \tilde{b}^{\prime}$ be parameters interpreted by $l$-prime elements $a$ and $b$ in $G T_{+} \cap B T$ and $a^{\prime}$ and $b^{\prime}$ in $G T_{-} \cap B T$.

There are formulas of $L \cup\left\{\tilde{e}, \tilde{a}, \tilde{b}, \tilde{a}^{\prime}, \tilde{b}^{\prime}\right\}$ which interpret:

(1) the chain $T_{G L}$ if $S_{a}<S_{b}$ and $S_{a^{\prime}}<S_{b^{\prime}}$,

(2) the chain $T_{G R}^{*}$ if $S_{b}<S_{a}$ and $S_{b^{\prime}}<S_{a^{\prime}}$.

In each case, if $s$ is an element of the interpretation of the chain and $f$ an $l$-prime element of bounded support, " $s \in{\overline{S_{f}}}_{f}$ " is equivalent to a formula of $L \cup\left\{\tilde{e}, \tilde{a}, \tilde{s}^{\prime}, \tilde{b^{\prime}}\right\}$, once the chain and its interpretation are identified in a natural way. 
Proof. Take $a$ and $b l$-primes in $G T_{+} \cap B T$ with $S_{a}<S_{b}$. We can interpret $T_{G L}$ in $G T_{+}$with techniques similar to those of the proof of Theorem 1: Let $D_{+}, O_{+}, E_{+}$ be formulas of $L \cup\{\tilde{e}, \tilde{a}, \tilde{b}\}$ such that:

$D_{+}$defines $G T_{+} \cap B T$ (Fact 5).

$O_{+}(u, v, a, b) \leftrightarrow \forall w>e\left(S_{u}<_{a, b} S_{w} \rightarrow S_{v}<_{a, b} S_{w}\right)$ (Fact 6).

$E_{+}(u, v, a, b) \leftrightarrow O_{+}(u, v, a, b) \wedge O_{+}(v, u, a, b)$.

The quotient set $D_{+} / E_{+}$with predicate $O_{+}$is in a natural way identified with the chain $T_{G L}$ when, for any $s$ in $D_{+} / E_{+}$and any $g$ in $G T_{+} \cap B T$, we let $s=l_{g}$ if $g$ belongs to $s$. For any $f$ and $g$ in $G T_{+} \cap B T, l_{f}=l_{g}$ is equivalent to $E(f, g, a, b)$.

Let $g$ be any element in $G T_{+} \cap B T-\{e\}$ and $f$ be any $l$-prime in $G T_{+} \cap B T$;

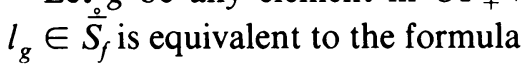

$$
\exists u\left(e<u \leqslant f \wedge E_{+}(u, g, a, b) \wedge \neg E_{+}(f, g, a, b)\right)
$$

since if $l_{g} \in{\stackrel{\circ}{S_{f}}}_{\text {, then }} f \wedge g \leqslant f$ and $\neg E_{+}(f, g, a, b)$ is satisfied, so the formula is satisfied.

If the formula is satisfied, then for some $u \leqslant f$ we have $S_{u} \subseteq S_{f}$, so $r_{f}<l_{u} \leqslant l_{f}$, with $l_{u}=l_{g}$ and $l_{f} \neq l_{g}$, hence $r_{f}<l_{g}<l_{f}$ and $l_{g} \in \dot{S}_{f}$.

Clearly, if $a$ and $b$ are l-primes in $G T_{+} \cap B T$ with $S_{b}<S_{a}, D_{+} / E_{+}$must be identified with $T_{G R}^{*}$ and the above formula is equivalent to $r_{g} \in{\stackrel{\circ}{S_{f}}}_{\text {. }}$.

Now take $a^{\prime}$ and $b^{\prime} l$-primes in $G T_{-} \cap B T$ with $a^{\prime} \wedge b^{\prime}=e$. In a similar way to what was done above, we can find formulas $D_{-}, E_{-}, \bar{O}_{-}$of $L \cup\left\{\tilde{e}, \tilde{a}^{\prime}, \tilde{b}^{\prime}\right\}$ interpreting one of $T_{G L}$ (if $S_{a^{\prime}}<S_{b^{\prime}}$ ) or $T_{G R}^{*}$ (if $S_{b^{\prime}}<S_{a^{\prime}}$ ) and a formula equivalent to $s_{g} \in \stackrel{\circ}{S_{f}}$ for any $s_{g}=l_{g}$ or $r_{g}$ in the interpretation and any $l$-prime $f$ in $G T_{-} \cap B T$.

If $a, b, a^{\prime}, b^{\prime}$ are any $l$-primes with $a$ and $b$ in $G T_{+} \cap B T$ and $a^{\prime}$ and $b^{\prime}$ in $G T_{-} \cap B T$, let $s_{g}$ be any element of $D_{+} / E_{+}$and $s_{h}$ be any element of $D_{-} / E_{-}$. Clearly, $s_{g}=s_{h}$ is equivalent to the following formula $E_{+-}$of $L \cup\left\{\tilde{e}, \tilde{a_{g}} \tilde{b}, \tilde{a}^{\prime}, \tilde{b}^{\prime}\right\}$ : $E_{+\bar{\sigma}_{\underline{o}}}\left(f, g, a, b, a^{\prime}, b^{\prime}\right) \leftrightarrow \forall u \in B T$ with $u \underline{\vee} e$ and $u \wedge e l$-primes $\left(s_{g} \in \ddot{S}_{u \underline{v} e} \rightarrow \neg s_{h}\right.$ $\left.\in \bar{S}_{u \wedge e}\right)$.

Hence:

(a) For any $l$-primes $f$ and $g, f$ in $G T_{+} \cap B T$ and $g$ in $G T_{-} \cap B T, \stackrel{\circ}{\bar{S}_{f}} \cap \stackrel{\circ}{\bar{S}_{g}} \neq \varnothing$ is equivalent to the following formula of $L \cup\{\tilde{e}\}: \exists u, v, u^{\prime}, v^{\prime} l$-primes in $B T$ with $u \wedge \boldsymbol{v}=e$ and $u^{\prime} \underline{\vee} v^{\prime}=e, \exists s_{w}$ in $D_{+} / E_{+}, \exists s_{w^{\prime}}$ in

$$
D_{-} / E_{-}\left(E_{+-}\left(w, w^{\prime}, u, v, u^{\prime}, v^{\prime}\right) \wedge s_{w} \in{\stackrel{\circ}{S_{g}}}^{\prime} \wedge s_{w^{\prime}} \in \stackrel{\circ}{S_{f}}\right) \text {, }
$$

where for any $l$-primes $f$ and $g$ both in $G T_{+} \cap B T$ (or both in $G T_{-} \cap B T$ ) ${\stackrel{\circ}{S_{f}}}_{\bar{S}_{g}} \neq \varnothing$ is equivalent to $f \wedge g \neq e($ or $f \underline{\vee} g \neq e)$.

So, for any $l$-primes $f$ and $g$ in $G T \cap B T, \stackrel{\circ}{S_{f}} \cap \stackrel{\circ}{S_{g}} \neq \varnothing$ is equivalent to a formula of $L \cup\{\tilde{e}\}$, and, for any $l$-primes $f, g$ and $h$ in $G T \cap B T$, " $S_{g}$ is between $S_{f}$ and $S_{h}$ " is equivalent to:

$$
\forall u l \text {-prime in } G T \cap B T\left(\left(\stackrel{\circ}{S_{u}} \cap \stackrel{\circ}{S_{f}} \neq \varnothing \wedge \stackrel{\circ}{S_{u}} \cap \stackrel{\circ}{S_{h}} \neq \varnothing\right) \rightarrow \stackrel{\circ}{S_{u}} \cap \stackrel{\circ}{S_{g}} \neq \varnothing\right)
$$

(compare with Fact 4), which achieves the proof of (a).

(b) If $S_{a}<S_{b}$ and $S_{a^{\prime}}<S_{b^{\prime}}, D_{+} / E_{+}$and $D_{-} / E_{-}$are two copies of $T_{G L}$ which can be identified into one by the formula $E_{+-}$. For any $s$ in this new copy of $T_{G L}$ and 
any $f$ l-prime in $G T \cap B T$, " $s \in \stackrel{\circ}{S_{f}}$ " is then clearly equivalent to a formula of $L \cup\left\{\tilde{e}, \tilde{a}, \tilde{b}, \tilde{a}^{\prime}, \tilde{b^{\prime}}\right\}$, which achieves the proof of (b), case 1 . Case 2 is dual.

Fact 8. We can find two subsets $P_{1}$ and $P_{2}$ of $G T$ such that $P=P_{1} \cup P_{2}$ is definable with $L \cup\{\tilde{e}\}\left(P_{1}\right.$ and $P_{2}$ generally not definable with $\left.L \cup\{\tilde{e}\}\right)$, and formulas of $L \cup\{\tilde{e}, \tilde{p}\}$ which interpret:

(1) the chain $T_{G L}$, if $\tilde{p}$ is interpreted by any $p \in P_{1}$,

(2) the chain $T_{G R}^{*}$, if $\tilde{p}$ is interpreted by any $p \in P_{2}$.

In both cases, if $s$ is an element of the interpretation of the chain and $f$ an $l$-prime element of bounded support, $s \in \stackrel{\circ}{S_{f}}$ is a formula of $L \cup\{\tilde{e}, \tilde{p}\}$, once the chain and its interpretation are identified in a natural way.

We shall prove Fact 8 together with the following remark, which we will use for Corollary 2-5.

Remark. The sets $P_{1}$ and $P_{2}$ of Fact 8 can be chosen such that $P_{2}=P_{1}^{-1}$, where $P_{1}^{-1}=\left\{f^{-1} ; f \in P_{1}\right\}$.

Proof. Using Fact 7, it will be enough to find a subset $P$ of $G T$, definable in $L \cup\{\tilde{e}\}$, such that $P=P^{-1}$, and formulas of $L \cup\{\tilde{e}, \tilde{p}\}$ defining four $l$-primes $a, b, a^{\prime}, b^{\prime}$ in $G T_{+} \cap B T$, such that, for any $p$ in $P$ : either $S_{a}<S_{b}<S_{a^{\prime}}<S_{b^{\prime}}$ when $\tilde{p}$ is interpreted by $p$ and $S_{b^{\prime}}<S_{a^{\prime}}<S_{b}<S_{a}$ when $\tilde{p}$ is interpreted by $p^{-1}$ or conversely.

So, for any $p$ in $G T$, let $P(p)$ be the formula of $L \cup\{e\}$ described below:

$P(p) \leftrightarrow\left\{\begin{array}{l}\exists u, v, u^{\prime}, v^{\prime} l \text {-primes in } G T \cap B T \text { such that } u \wedge v=e, u \underline{\vee} v=p \underline{\vee} e \\ u^{\prime} \underline{\vee} v^{\prime}=e, u^{\prime} \wedge v^{\prime}=p \wedge e \text { and none of } S_{u} \text { and } S_{v} \text { is between } S_{u^{\prime}} \text { and } S_{v^{\prime}}\end{array}\right.$

$P$ defines the set of elements of $G T \cap B T$ which are a product of four $l$-primes of pairwise disjoint supports, among which two are in $G T_{+}$and have supports both less or both greater than the supports of the two others, which are in $G T_{-}$. Clearly $P=P^{-1}$.

For any $p$ in $P$, let $a, b, a^{\prime}, b^{\prime}$ be defined with $L \cup\{\tilde{e}, \tilde{p}\}$ where $\tilde{p}$ is interpreted by $p$ as below:

$a$ is a positive $l$-prime restriction of $p$ and its support is not between the supports of a positive and a negative restriction of $p$ (where " $u$ is a positive restriction of $p$ " is clearly equivalent to " $\exists v((u \wedge v=e) \wedge(u \underline{\vee} v=p \underline{\vee} e))$ " and " $u$ is a negative restriction of $p$ " dually).

$b$ is the positive $l$-prime restriction of $p$ which is not $a$.

$a^{\prime}$ is a negative $l$-prime restriction of $p$ and its support is between the supports of a positive and a negative restriction of $p$.

$b^{\prime}$ is the negative $l$-prime restriction of $p$ which is not $a^{\prime}$.

Now, for any $p$ in $P$ we have either $S_{a}<S_{b}<S_{a^{\prime}}<S_{b^{\prime}}$ when $\tilde{p}$ is interpreted by $p$ and $S_{b^{\prime}}<S_{a^{\prime}}<S_{b}<S_{a}$ when $\tilde{p}$ is interpreted by $p^{-1}$ or conversely.

Fact 9. Let $S$ be the interpretation of one of $T_{G L}$ or $T_{G R}^{*}$ within $L \cup\{\tilde{e}, \tilde{p}\}$ as in Fact 8. We can find a formula of $L \cup\{\tilde{e}, \tilde{p}\}$ which is satisfied by any $f$ in $G T$ and any $s$ and $t$ in $S$ if and only if either $f(s)=t$ and $S$ interprets $T_{G L}$ or $f(t)=s$ and $S$ interprets $T_{G R}^{*}$. 
Proof. Take $s$ and $t$ in $S$ such that the interpretation of $s \leqslant t$ is satisfied in $G T$. The chain $\bar{T}$ satisfies $s \leqslant t$ if $S$ is (identified with) $T_{G L}$ and $t \leqslant s$ if $S$ is (identified with) $T_{G R}^{*}$.

Assume $S$ is $T_{G L}$ and let $f$ be any element of $G T_{+}$. We claim that $f(s) \leqslant t$ is equivalent to the following formula $F(f, s, t)$ of $L \cup\{\tilde{e}, \tilde{p}\}$ :

$$
\begin{aligned}
F(f, s, t) & \leftrightarrow \forall u l \text {-prime in } G T_{+} \cap B T\left(s \text { and } t \in \dot{\circ}_{u}\right) \\
& \rightarrow\left(\exists v\left(S_{v} \subseteq \bar{S}_{u} \text { and } \neg(v \leqslant f)\right)\right) .
\end{aligned}
$$

Assume $f(s) \leqslant t$ and let $u$ be an l-prime of $G T$ such that $s$ and $t \in \check{S}_{u}$. Since

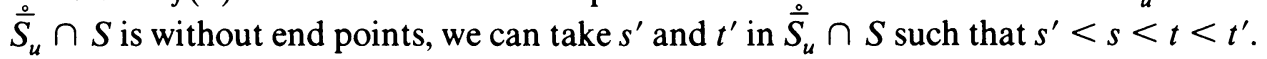

By 0-4 transitivity of $G T$ on $T$, we can find an element $v$ of $G T$ such that $S_{v} \subseteq\left[s^{\prime}, t^{\prime}\right] \subseteq \bar{S}_{u}$ and $v(s)>t$. Now $v(s) \geqslant t>f(s)$ so $v$ is not less than $f$ and $F(f, s, t)$ is satisfied.

Conversely, assume $f(s)>t$ and let $t^{\prime}$ be some element of $S$ such that $t<f\left(t^{\prime}\right)<$ $f(s)$.

We have $t^{\prime}<s \leqslant t<f\left(t^{\prime}\right)$ and so, by $0-4$ transitivity of $G T$ on $T$, we can pick some $l$-prime $u$ in $G T_{+} \cap B T$ such that $s$ and $t$ are in ${\stackrel{\circ}{S_{u}}}_{u}$ and $S_{u} \subseteq\left[t^{\prime}, f(t)\right]$.

Now, for any $v$ such that $S_{v} \subseteq \bar{S}_{u}$, we have $S_{v} \subseteq\left[t^{\prime}, f\left(t^{\prime}\right)\right]$, and so, for any $s^{\prime}$ in $S_{v}$, $v\left(s^{\prime}\right) \leqslant f\left(t^{\prime}\right) \leqslant f\left(s^{\prime}\right)$ so $v$ is less than $f$ and $F(f, s, t)$ is not satisfied.

It is now clear that if $S$ is $T_{G L}$ and $f$ is any element of $G T_{+}, f(s)=t$ is equivalent to

$$
F(f, s, t) \wedge \forall u \in S(F(f, s, u) \rightarrow t \leqslant u \text { in } S) .
$$

If $S$ is $T_{G R}^{*}$, to see that this same formula is equivalent to $f(t)=s, f$ in $G T_{+}$, it is enough to notice that $F(f, s, t)$ and $F(f, t, s)$ are equivalent.

The interpretation of the action of elements in $G T_{-}$on $s$ and $t$ can be done in a dual way. The interpretation of the action of any $f$ in $G T$ will then follow from the interpretation of $f \vee e$ and $f \wedge e$.

Fact 10. Theorem 2 is proved.

Proof. Facts 8 and 9 prove Theorem 2 when the parameter $\tilde{e}$ is interpreted by $e$. Since for any element $f$ in $G T$ the group-translation $g \mapsto f g$ is a lattice isomorphism with the image of $e=f$, the result is independent of the interpretation of parameter $\tilde{e}$.

The following result, similar to Corollary $1-5$, will have a similar proof, left to the reader.

COROLLARY 2-4. If GT and HS are 0-2 transitive l-p-groups with l-prime elements of bounded support such that:

(i) $\bar{T}=T_{G L}=T_{G R}$,

(ii) $\bar{S}=S_{G L}=S_{G R}$, and

(iii) for some integer $n, G T$ has at least $n$ orbits in $\bar{T}$ and HS has less than $n$ orbits in $\bar{S}$

then GT and HS are not elementary equivalent as lattices. 
The characterization of lattice isomorphisms will use Corollary 1-6 and Theorem 2.

COROLlaRY 2-5. Let GT and HS be 0-2 transitive l-p-groups with l-prime elements of bounded support, then any lattice isomorphism $\Phi$ from GT onto HS is of one of the forms:

(i) $\Phi(f)=k f h^{-1}$ for any $f$ in $G T$ where $k$ and $h$ are order isomorphisms from $T$ onto some orbit of $H S$ in $\bar{S}$.

(ii) $\Phi(f)=k^{\prime} f^{-1} h^{\prime-1}$ for any $f$ in $G T$ where $k^{\prime}$ and $h^{\prime}$ are anti-order isomorphisms from $T$ onto some orbit of $H S$ in $\bar{S}$.

Proof. Let $\Phi$ be a lattice isomorphism from $G T$ onto $H S$ as in the hypothesis of Corollary 2-5.

Let $e_{G}$ be the identity of $G T, e_{H}$ the identity of $H S$ and $\Phi^{\prime}$ the map such that

$$
\Phi^{\prime}(f)=\left(\Phi\left(e_{G}\right)\right)^{-1} \Phi(f) \text { for any } f \text { in } G T \text {. }
$$

Clearly $\Phi^{\prime}$ is a lattice isomorphism from $G T$ onto $H S$ such that $\Phi^{\prime}\left(e_{G}\right)=e_{H}$.

Let $p$ be an element satisfying formula $P$ of Theorem 2 in $G T$, and choose formulas of the lattice language, with parameters interpreted in $G T$ by $e_{G}$ and $p$, which interpret $T_{G L}$ in $G T$.

The same formulas with parameters interpreted in $H S$ by $\Phi\left(e_{G}\right)=e_{H}$ and $\Phi(p)$ will interpret in $H S$ one of:

(1) the full structure $H S \cup S_{G L}$,

(2) the full structure $H_{*} S \cup S_{G L}^{*}$.

In case (1) $\Phi^{\prime}$ is a group and lattice isomorphism from $G T$ onto $H S$. Hence, from Corollary 1-6 and the fact that $\Phi^{\prime}$ cannot reverse order, we have $\Phi^{\prime}(f)=h f h^{-1}$ for any $f$ in $G T$ where $h$ is an order isomorphism from $T$ onto some orbit of $H S$ in $\bar{S}$, hence $\Phi(f)=k f h^{-1}$ for any $f$ in $G T$ where $k=\Phi\left(e_{G}\right) h$ and $h$ are order isomorphisms from $T$ onto some orbit of $H S$ in $\bar{S}$. This is case (i) of Corollary 2-5.

In case (2) $\Phi^{\prime}$ is an anti-group and a lattice isomorphism from $G T$ onto $H S$. Let $\Phi^{\prime \prime}$ be the map such that $\Phi^{\prime \prime}(f)=\left(\Phi^{\prime}(f)\right)^{-1}$ for any $f$ in $G T$.

$\Phi^{\prime \prime}$ is a group and anti-lattice automorphism from $G T$ onto $H S$. Hence, from Corollary 1-6 and the fact that $\Phi^{\prime \prime}(f)$ cannot preserve order, we get $\Phi^{\prime \prime}(f)=h^{\prime} f h^{\prime-1}$ for any $f$ in $G T$ where $h$ is an anti-order isomorphism from $T$ onto some orbit of $H S$ in $\bar{S}$, and $\Phi(f)=k^{\prime} f^{-1} h^{\prime-1}$ for any $f$ in $G T$ where $k^{\prime}=\Phi\left(e_{G}\right) h^{\prime}$ and $h^{\prime}$ are anti-order isomorphisms from $T$ onto some orbit of $H S$ in $\bar{S}$. This is case (ii) of Corollary 2-5.

We can also have a similar result to Corollary $1-7$, with a similar proof, left to the reader:

COROLlaRY 2-6. The class of lattices which admit a structure of 0-2 transitive l-p-group with an l-prime element of bounded support is the class of models of one formula.

Note that, from Corollaries 1-7 and 1-2, the class of groups which admit a structure of 0-2 transitive $l$-p-group with an $l$-prime element of bounded support is also the class of models of one formula. From Corollaries 2-6 and 2-1, if $F$ is any 
formula of the language of full structures, the two classes of lattices $G T$ which admit a structure of $0-2$ transitive $l$-p-group with an $l$-prime element of bounded support and are such that:

(1) $F$ is satisfied in both $G T \cup T_{G L}$ and $G_{*} T \cup T_{G R}^{*}$, or

(2) $F$ is satisfied in one of $G T \cup T_{G L}$ and $G_{*} T \cup T_{G R}^{*}$, are also classes of models of one formula.

\section{REFERENCES}

1. A. M. W. Glass, Ordered permutation groups, London Math. Soc. Lecture Notes Series, Cambridge Univ. Press, Cambridge, 1981.

2. A. M. W. Glass, Y. Gurevich, W. C. Holland and S. Shelah, Rigid homogeneous chains, Math. Proc. Cambridge Philos. Soc. 89 (1981), 7-17.

3. Y. Gurevich and W. C. Holland, Recognizing the real line, Trans. Amer. Math. Soc. 265 (1981), $527-534$.

4. W. C. Holland, Outer automorphisms of 0-permutation groups, Edinburgh Math. Soc. 19 (1976), $331-334$.

5. M. Jambu-Giraudet, Théorie des modèles de groupes d'automorphismes d'ensembles totalement ordonnés, Thèse de troisième cycle, Univ. Paris VII, 1979.

6. Théorie des modèles de groupes d'automorphismes d'ensembles totalement ordonnés 2 homogènes, C. R. Acad. Sci. Paris Sér. A-B 290 (1980), 1037-1039.

7. L L L L L L theory of some lattice-ordered groups, Abstracts Amer. Math. Soc. 1 (1980), 477. Abstract 80T-A154.

8. S. H. McCleary, Groups of homeomorphisms with manageable automorphism group, Comm. Algebra 6 (1978), 497-528.

9. B. Rabinovich, On linearly ordered sets with 2-transitive groups of automorphisms, Vesci Akad. Navuk BSSR Ser. Fìz.-Mat. Navuk 6 (1975), 10-17. (Russian)

32, Rue de la Reunion, 75020 Paris, XX ${ }^{\mathrm{e}}$, France 